\title{
Validated UPLC-MS/MS method for quantification of fruquintinib in rat plasma and its application to pharmacokinetic study
}

This article was published in the following Dove Press journal:

Drug Design, Development and Therapy

\author{
Yi-Bin Mei ${ }^{1, *}$ \\ Shun-Bin Luo ${ }^{2, *}$ \\ Ling-Yan Ye' \\ Qiang Zhang ${ }^{3}$ \\ Jing Guo ${ }^{4}$ \\ Xiang-Jun Qiu ${ }^{5}$ \\ Sai-Li Xie ${ }^{6}$ \\ 'Department of Cardiology, The People's \\ Hospital of Lishui, Lishui, Zhejiang 323000 , \\ People's Republic of China; ${ }^{2}$ Department \\ of Clinical Pharmacy, The People's Hospital \\ of Lishui, Lishui, Zhejiang 323000, People's \\ Republic of China; ${ }^{3}$ Department of Clinical \\ Laboratory, The People's Hospital of \\ Lishui, Lishui, Zhejiang 323000, People's \\ Republic of China; ${ }^{4}$ Department of \\ Regional Medical Union, The People's \\ Hospital of Lishui, Lishui, Zhejiang 323000, \\ People's Republic of China; ${ }^{5}$ Department \\ of pharmacology, Medical College of \\ Henan University of Science and \\ Technology, Luoyang 471003, People's \\ Republic of China; ${ }^{6}$ Department of \\ Ultrasonic imaging, The First Affiliated \\ Hospital of Wenzhou Medical University, \\ Wenzhou, Zhejiang 325035, People's \\ Republic of China \\ *These authors contributed equally to this \\ work
}

Correspondence: Xiang-Jun Qiu Department of pharmacology, Medical College of Henan University of Science and Technology, No. 6 Anhui Road, Luoyang 471003, People's Republic of China

Email lyxiangjun@I26.com

Sai-Li Xie

Department of Ultrasonic imaging, The First Affiliated Hospital of Wenzhou

Medical University, Shangcai village,

Nanbaixiang town, Ouhai district,

Wenzhou, Zhejiang 325000, People's

Republic of China

Email xiesailiI988@I63.com

\begin{abstract}
A new, simple, and sensitive ultraperformance liquid chromatography-tandem mass spectrometry (UPLC-MS/MS) method for quantification of fruquintinib was established to assess the pharmacokinetics of fruquintinib in the rat. The internal standard working solution was added to the plasma sample for extraction before analysis. The Acquity UPLC BEH C18 chromatography column $(2.1 \mathrm{~mm} \times 50 \mathrm{~mm}, 1.7 \mu \mathrm{m})$ was used to separated analytes under gradient elution using acetonitrile and $0.1 \%$ formic acid as the mobile phase. Positive multiple reaction monitoring modes were chosen to detect fruquintinib and diazepam (IS). The precursor-to-product ion transitions were $394.2 \rightarrow 363.2$ for fruquintinib and $\mathrm{m} / \mathrm{z} 285 \rightarrow$ 154 for IS. The current method was linear over the concentration range of $1.0-1000 \mathrm{ng} / \mathrm{mL}$ for fruquintinib with a correlation coefficient of 0.9992 or better. The matrix effect of fruquintinib and IS was acceptable under the current method. The intra- and interday precision (RSD\%) and accuracy (RE\%) were within $11.9 \%$ and $\pm 13.7 \%$, respectively. The recovery, stability, and sensitivity were validated according to the United States Food and Drug Administration (FDA) regulations for bioanalytical method validation. The analytical method had been validated and applied to a pharmacokinetic study of fruquintinib in rat.
\end{abstract}

Keywords: fruquintinib, UPLC-MS/MS, rat plasma, pharmacokinetics

\section{Introduction}

As an important hallmark of cancer development and progression, angiogenesis plays a major role in vascular remodeling, cell proliferation, cancer cell dissemination, and metastasis. ${ }^{1-4}$ Vascular endothelial growth factor (VEGF) secreted by tumors promotes the formation of the angiogenesis. The angiogeneses around the tumor supplied the blood flow, oxygen, and nutrients to the tumor at an advanced stage. The anti-cancer therapies targeting VEGF/vascular endothelial growth factor receptors (VEGFR) axis included neutralization of VEGF or VEGFR by monoclonal antibodies and blockage of VEGFR kinase activity with small molecule inhibitors. ${ }^{5}$ Due to its long half-life and high specificity, bevacizumab can sustain target inhibition and also combine with other chemotherapies. It limits bevacizumab clinical use or patients' access because of intravenous dosing, autoimmune diseases after long-term treatment, immunogenicity, and especially high cost. ${ }^{6}$ Therefore, an active small molecule VEGFR inhibitor by oral administration combined with chemotherapies to achieve sustained target inhibition and avoid the limitation of antibodies is highly desirable. 
Small-molecule VEGFR inhibitors include first-generation sunitinib, ${ }^{7}$ regorafenib, ${ }^{8,9}$ sorafenib ${ }^{10}$ and pazopanib; ${ }^{11}$ second-generation tivozanib ${ }^{12}$ and axitinib. ${ }^{13}$ The less selective of these inhibitors limited their clinical use. New-generation tyrosine kinase inhibitors (TKIs) include icotinib, ${ }^{14}$ apatinib, ${ }^{15}$ famitinib, ${ }^{16}$ flumatinb, ${ }^{17}$ allitinib, ${ }^{18}$ fruquintinib, ${ }^{19}$ and selatinib, ${ }^{20}$ among which icotinib and apatinib have been approved in China, while others are in clinical trials. Fruquintinib (6-(6, 7-dimethoxyquinazolin-4-yloxy) - N,2dimethylbenzofuran-3-carboxamide, Figure 1A) developed by Hutchison MediPharma Ltd. (Shanghai, China) had been studied in Phase II and Phase III clinical trial for non-small-cell lung cancer ("NSCLC") and colorectal cancer (CRC) and demonstrated highly potent efficacy and safety profile in vitro and in vivo. ${ }^{19,21-24}$ In 2018, fruquintinib was approved in China for treatment of CRC. It is an oral TKI with a highly selective small-molecule inhibitor of VEGFR-1, -2, and -3, thus inhibiting endothelial cell proliferation and migration. ${ }^{2,19,21}$

When more than two prior systemic antineoplastic therapies have failed to treatment metastatic CRC patients, fruquintinib will be used in these patients. To the best of our knowledge, there are several LC-MS/MS methods reported for the quantitative bioanalysis of fruquintinib in plasma. ${ }^{21,25}$ However, these methods were not described in enough detail (eg, plasma extraction procedure, chromatography conditions, parameters of the method, etc.) for duplication in other laboratories. Furthermore, the time of sample collection was too short, and the plasma concentration of the parent compound was still above the minimum effective concentration or lower limit of quantitation (LLOQ). Therefore, the current study is aimed to develop a new and sensitive ultra performance liquid chromatography-tandem mass spectrometry (UPLC-MS/MS) method for the quantification of fruquintinib and assess the pharmacokinetics of fruquintinib with sufficient time to metabolize in the rat.

\section{Materials and methods}

\section{Chemicals and reagents}

Fruquintinib (CAS NO: 1194506-26-7) was purchased from Shanghai Boylechem Co., Ltd (Songjiang District, Shanghai, China); diazepam (CAS NO: 65854-76-4) as internal standard (IS) (Figure 1B) and formic acid (CAS:64-18-6) were provided by Sigma-Aldrich Company (St. Louis, MO, USA); all materials were suitable for use in LC/MS or UPLC/MS laboratory testing by spiking into the matrix of choices such as urine or plasma. Acetonitrile with LC grade was supplied from Merck Company (Darmstadt, Germany), and the other reagents were all analytical grade. The water used in the laboratory was prepared by Wahaha pure water which filtered through a 0.45 $\mu \mathrm{m}$ filter. $0.1 \%$ formic acid was prepared by $1 \mathrm{~mL}$ formic acid diluted with the water to $1 \mathrm{~L}$.

\section{Analytical system}

The Acquity UPLC BEH C18 chromatography column $(2.1 \mathrm{~mm} \times 50 \mathrm{~mm}, 1.7 \mu \mathrm{m})$ was used to separated analytes

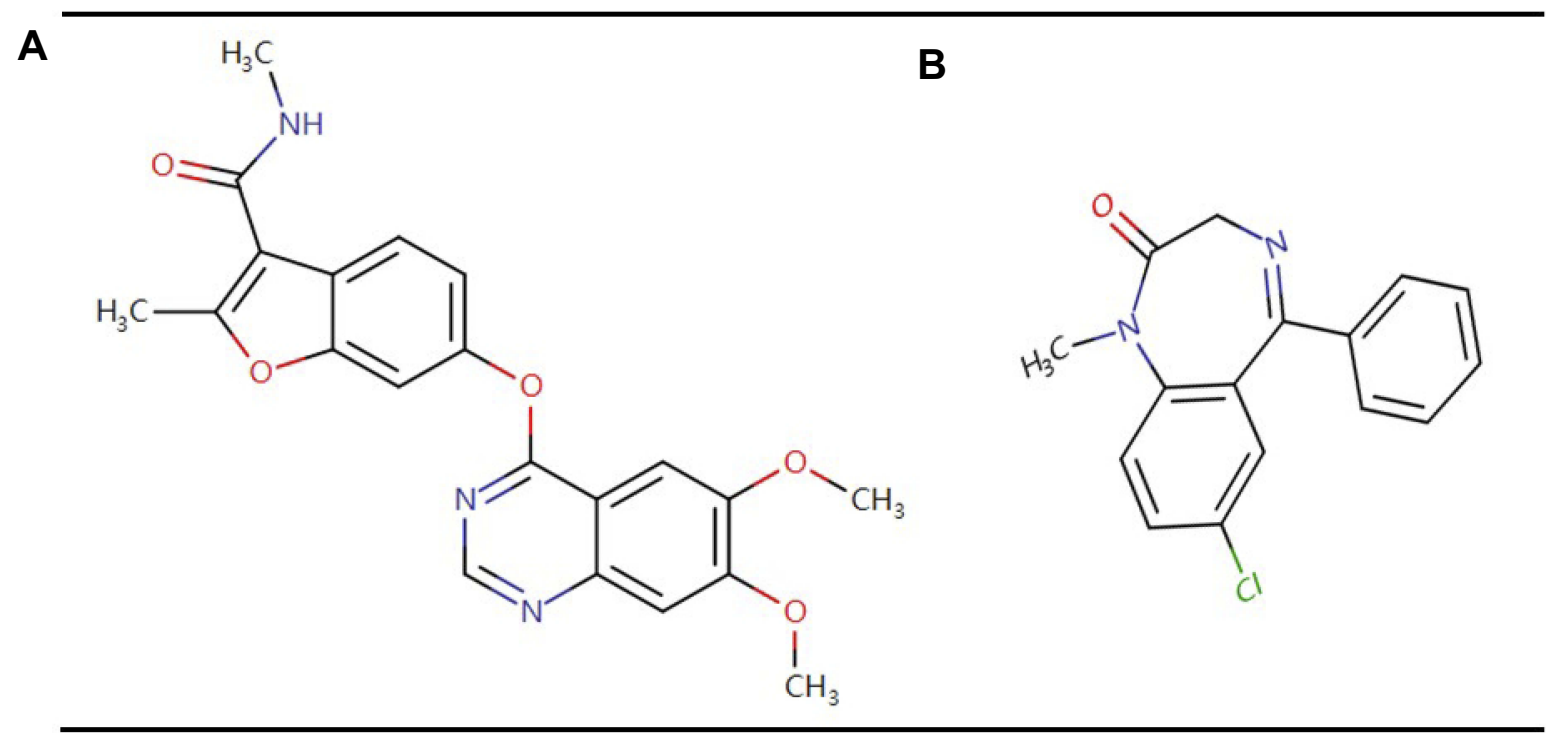

Fruquintinib

Diazepam

Figure I The chemical structures of the analytes in the present study. (A) fruquintinib; (B) diazepam. 
under gradient elution using acetonitrile (mobile phase A) and $0.1 \%$ formic acid (mobile phase $\mathrm{B}$ ) as the mobile phase. The gradient elution program was as follows: $0-0.5 \mathrm{mins}(90 \% \mathrm{~A})$, 0.5-1 mins (90-10\% A), 1.0-2.0 mins (10\% A), 2.0-2.1 mins (10-90\% A), and 2.1-3.0 mins (90\% A). The overall chromatographic run time was $3.0 \mathrm{~min}$ and the flow rate of the method was set at $0.30 \mathrm{~mL} / \mathrm{min}$. Mass spectrometric detection was performed on a XEVO TQD triple quadrupole mass spectrometer equipped with an electrospray ionization (ESI) source (Waters Corp., Milford, MA, USA). The analytical method used positive multiple reaction monitoring mode to detect fruquintinib and diazepam (IS). The precursor-to-product ion transitions were $394.2 \rightarrow 363.2$ for fruquintinib and $\mathrm{m} / \mathrm{z} 285$ $\rightarrow 154$ for IS (desolvation temperature $500^{\circ} \mathrm{C}$, desolvation gas flow rate $600 \mathrm{~L} / \mathrm{h}$, the argon flow rate $150 \mathrm{~L} / \mathrm{h}$, collision 7.0 Bar). All data acquisition and instrument control were processed under the Masslynx 4.1 software (Waters Corp.).

\section{Preparation of standard solutions and quality control sample (QC)}

Fruquintinib or IS was dissolved in acetonitrile and prepared to a stock solution $(1 \mathrm{mg} / \mathrm{mL})$ which waws stored at $4^{\circ} \mathrm{C}$. The standard solutions were diluted to the stock solution with acetonitrile. Calibration curves standard solutions were prepared by adding blank plasma samples to the corresponding working solutions. The final concentrations of the calibration curves were as follows: 1.0, 5.0, $10,50,100,500$, and $1000 \mathrm{ng} / \mathrm{mL}$ for fruquintinib. The quality control sample (QC) solutions were prepared in the same manner to the following concentrations: $2.0,80$, and $800 \mathrm{ng} / \mathrm{mL}$ for fruquintinib. The above-prepared solutions were all stored at $-20^{\circ} \mathrm{C}$ until analysis.

\section{Plasma sample preparation}

$100 \mu \mathrm{L}$ thawed plasma sample was spiked with $200 \mu \mathrm{L}$ acetonitrile (IS $100 \mathrm{ng} / \mathrm{mL}$ ) and vortex mixed for $1.0 \mathrm{~min}$, and then centrifugation was done at $13,000 \mathrm{~g}$ for 10 mins. A clear supernatant $(6 \mu \mathrm{L})$ after centrifugation was injected into the analytical system for analysis.

\section{Method validation}

The UPLC-MS/MS method for quantification of fruquintinib in rat plasma and its application to pharmacokinetic study was validated according to the FDA guidelines. ${ }^{26}$

Blank rat plasma samples spiked with fruquintinib and IS were used to carry out selectivity. Specificity was evaluated by the interference at the retention time of fruquintinib and IS.

A weighted $\left(1 / x^{2}\right)$ least-squares linear regression was used to assess the linearity. The current method was linear over a range of $1.0-1000 \mathrm{ng} / \mathrm{mL}$ for fruquintinib with a correlation coefficient of 0.99 or better. A deviation of $\pm 15 \%$ for each back-calculated standard concentration was accepted, while it was $\pm 20 \%$ for LLOQ.

The precision and accuracy of our method were evaluated by analyzing 6 replicates at 2, 80, $800 \mathrm{ng} / \mathrm{mL}$ within 1 day or on 3 consecutive days. Precision was measured as a relative standard deviation ( $\mathrm{RSD} \%$ ), while the accuracy was a relative error $(\mathrm{RE} \%)$. A deviation of $\pm 15 \%$ for the accuracy and precision was accepted.

The recovery was calculated as the ratio of the peak area of the analyte in QCs to the post-protein precipitation at equivalent analyte concentrations (6 replicates at 2, 80, and $800 \mathrm{ng} / \mathrm{mL}$ ). The matrix effects of rat plasma were assessed by comparing the ratio of the analyte concentration in the post-extracted sample to the pure standard solution at equivalent concentrations.

The stability of fruquintinib at different conditions (room temperature for $12 \mathrm{hrs}, 4^{\circ} \mathrm{C}$ for $12 \mathrm{hrs},-80^{\circ} \mathrm{C}$ for 28 days, and three freeze-thaw cycles from $-20^{\circ} \mathrm{C}$ to room temperature) was evaluated by analyzing 5 replicates at three concentration levels $(2,80,800 \mathrm{ng} / \mathrm{mL})$. It could be permitted when the analyte stability was between $85 \%$ and $115 \%$.

\section{The pharmacokinetic study in the rat}

Fruquintinib dissolved in $0.5 \%$ sodium carboxymethyl cellulose $(1.0 \mathrm{mg} / \mathrm{mL})$ was orally given to eight young adult Sprague-Dawley rats (2 months, 180-220 g), and the volume of the suspension given to the animal was 1.0 $\mathrm{mL} / \mathrm{kg}$. The rat blood samples $(0.3 \mathrm{~mL})$ were taken via caudal vein at the following time points after drug administration: $0.33,0.67,1,1.5,2,3,4,6,9,12,24$, and $48 \mathrm{hrs}$. $100 \mu \mathrm{L}$ plasma was separated from whole blood after centrifuged at $10,000 \mathrm{~g}$ for $5 \mathrm{~min}$, and then stored at $-20^{\circ} \mathrm{C}$ before analysis. Approved by the Wenzhou Medical University Ethics Committee (SYXK 20150009), the study was performed under the National Institutes of Health Guide for the Care and Use of Laboratory Animals. Noncompartmental pharmacokinetic parameters were calculated for fruquintinib. ${ }^{27-29}$ The pharmacokinetic parameters of fruquintinib were noted directly by DAS (Drug and statistics) software (Version 2.0, 
Shanghai University of Traditional Chinese Medicine, China). ${ }^{30}$

\section{Results and discussion}

The development and optimization of the method

Although liquid extraction, protein precipitation, and solid-phase extraction have been commonly used to extract analytes from plasma or serum in many studies, there are not many pieces of research to study the method for determination fruquintinib so far. It reports that liquid chromatography-tandem mass spectrometry (LC-MS/MS) had been used to determine fruquintinib in rat plasma. ${ }^{16,21,25}$ Due to time saving, simplicity, low matrix effects and the comparative results that we did, we adopted UPLC-MS/MS and the protein precipitation. Meanwhile, UPLC-MS/MS was available in most laboratories at present. The proper combination of mobile phases helped to achieve peak symmetry, shorten analysis time and optimize ionization. When compared to methanol- $0.1 \%$ formic acid, acetonitrile- $0.1 \%$ formic acid with gradient elution could provide better effects and less matrix effects. The other parameters of MS/MS conditions and chromatography had also been made optimal at the same time.

\section{Selectivity and matrix effect}

Representative chromatograms are shown in Figure 2. No evident interference was found at the elution times: fruquintinib and IS were eluted at 1.40 and $1.59 \mathrm{~min}$, respectively.

The matrix effect of fruquintinib ranged from $87.4 \%$ to $95.6 \%$, while the IS was $102.4 \%$. Therefore, it was negligible for ion suppression or enhancement from rat plasma in the study.

\section{Calibration curve and sensitivity}

The current method was linear over a range of 1.0-1000 $\mathrm{ng} / \mathrm{mL}$ for fruquintinib with a correlation coefficient of 0.9992 , and the LLOQ was $1.0 \mathrm{ng} / \mathrm{mL}$ for fruquintinib. The calibration curve was $y=2.8493 x+7.1062(\mathrm{n}=3)$, where $y=$ the peak area ratio of fruquintinib to IS, $x=$ the plasma concentration of fruquintinib. The LLOQ with RSD $<11.9 \%$ and $\mathrm{RE} \leq 13.7 \%$ was acceptable in the present study.

\section{Precision, accuracy, and recovery}

The results in Table 1 represented the accuracies and precisions. The accuracies (RE\%) of the method was within $\pm 13.7 \%$, while the precisions (RSD\%) within $11.9 \%$. The results demonstrated that the UPLC-MS/MS method was suited for determination fruquintinib. The recovery by protein precipitation with acetonitrile was between $82.0 \%$ and $94.4 \%$.

\section{Stability}

The stability under different storage and process conditions (room temperature for $12 \mathrm{hrs}, 4^{\circ} \mathrm{C}$ for $12 \mathrm{hrs},-80^{\circ} \mathrm{C}$ for 28 days, and three freeze--thaw cycles from $-20^{\circ} \mathrm{C}$ to room temperature) was demonstrated and the results are summarized in Table 2 and found to be acceptable (within $\pm 15 \%$ ).

\section{Pharmacokinetic study of fruquintinib}

The current UPLC-MS/MS method was applied to the pharmacokinetic study after a single oral dose $(1.0 \mathrm{mg} / \mathrm{kg})$ of fruquintinib. The noncompartment model was used in the calculations of pharmacokinetic parameters. The pharmacokinetic parameters included $t_{1 / 2}, T_{\max }, \mathrm{CL} / \mathrm{F}, C_{\max }$, AUC and are shown in Table 3 . The mean plasma concentrationtime profile of fruquintinib after single-dose administration is presented in Figure 3.

Fruquintinib was absorbed rapidly after oral administration of $1.0 \mathrm{mg} / \mathrm{kg}$. $C_{\max }$ was reached at $4.25 \mathrm{hrs}$ after dosing, consistent with the previous study. ${ }^{25}$ Because of slow elimination and low tissue distribution, the mean $t_{1 / 2}$ of fruquintinib was about $3.34 \mathrm{hrs}$ in rat plasma. There were few studies to quantitate bioanalysis of fruquintinib in rat plasma. A study indicated that the terminal $t_{1 / 2}$ ranged from 1 to $2 \mathrm{hrs}$ in rat. ${ }^{25}$ But the time of sample collection was too short in that study, and the plasma concentration of parent compound was still above the minimum effective concentration or LLOQ. Meanwhile, the oral bioavailability of fruquintinib in the rat was 42 $53 \%$ and its plasma protein binding fraction is $88-95 \%$. The extents of CL and $\mathrm{V}_{\mathrm{z}}$ in each species were different, which might lead to the different $t_{1 / 2}$ profiles. The above facts could explain the data discrepancy in $C_{\max }(712.28 \mathrm{vs}$ $326.00 \mathrm{ng} / \mathrm{mL})$ and $t_{1 / 2}(3.34 \mathrm{vs} 1.40 \mathrm{hrs})$.

\section{Conclusion}

In summary, a fast, simple, sensitive, and accurate UPLC-MS/ MS method was established for quantitation of fruquintinib in 


\section{A}

MRM of 3 Channels ES+

$100 \quad 0.23,0.30,0.400 .68,0.74 \quad 1.151 .31,1.36 \quad 1.751 .82 \quad 2.13 \quad 394.2>363.2$ (Fruquintinib)

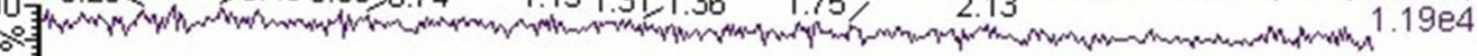

$\begin{array}{llllll}0.50 & 1.00 & 1.50 & 2.00 & 2.50 & 3.00\end{array}$

MRM of 3 Channels ES+

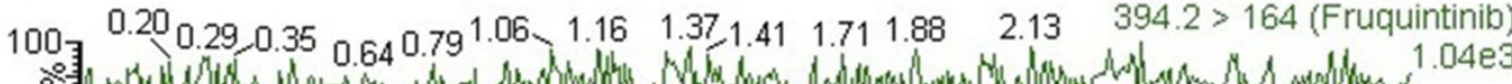
열. \begin{tabular}{llllll}
\hline & 0.50 & 1.00 & 1.50 & 1 & 1 \\
\hline
\end{tabular} MRM of 3 Channels ES+

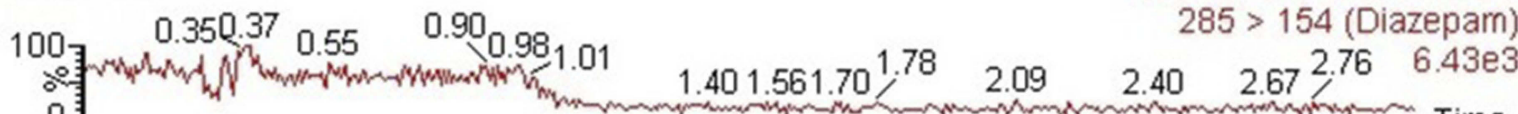
(1) Time $\begin{array}{llllll}0.50 & 1.00 & 1.50 & 2.00 & 2.50 & 3.00\end{array}$

\section{B}

MRM of 3 Channels ES+

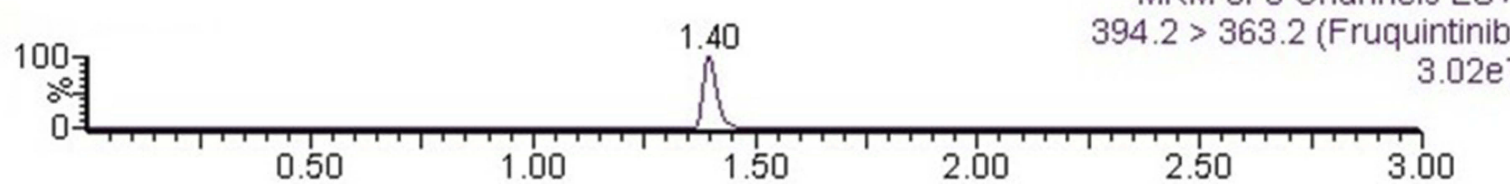

MRM of 3 Channels ES+
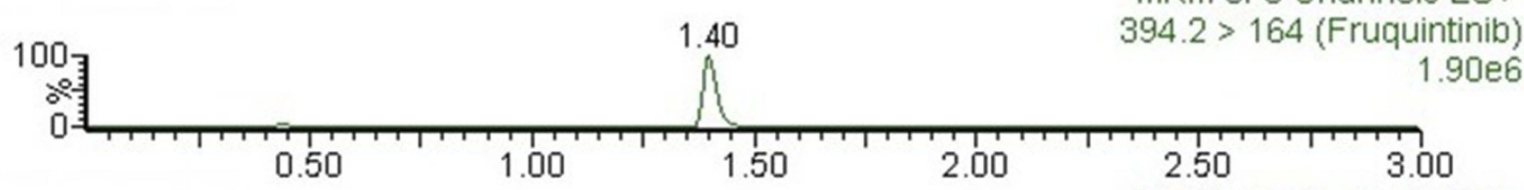

MRM of 3 Channels ES+

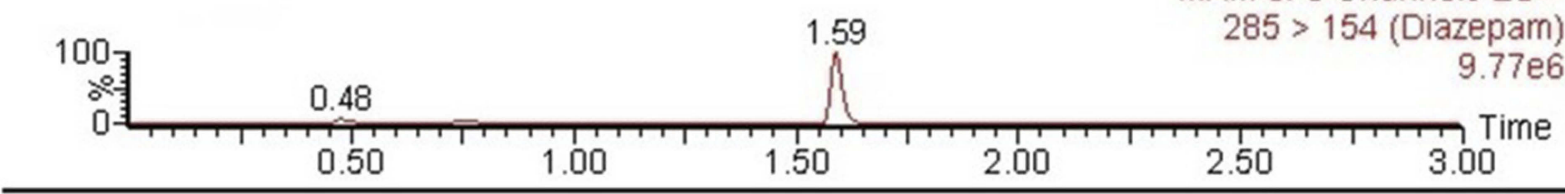

C

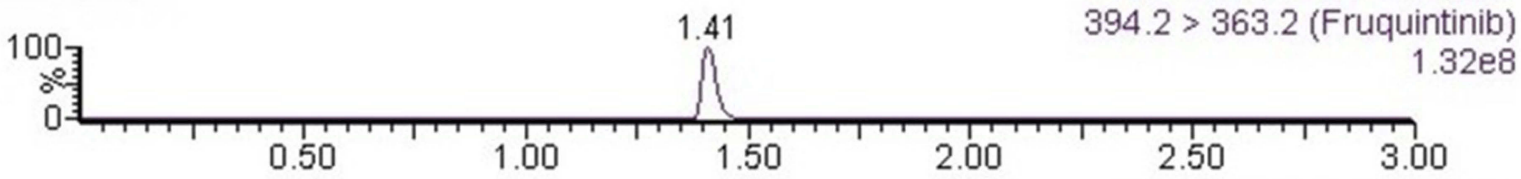

MRM of 3 Channels ES+

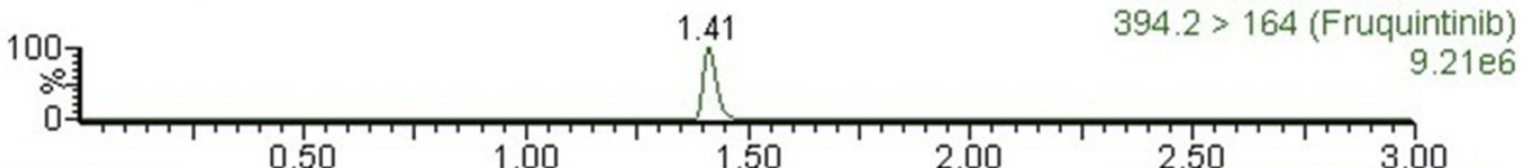

MRM of 3 Channels ES+

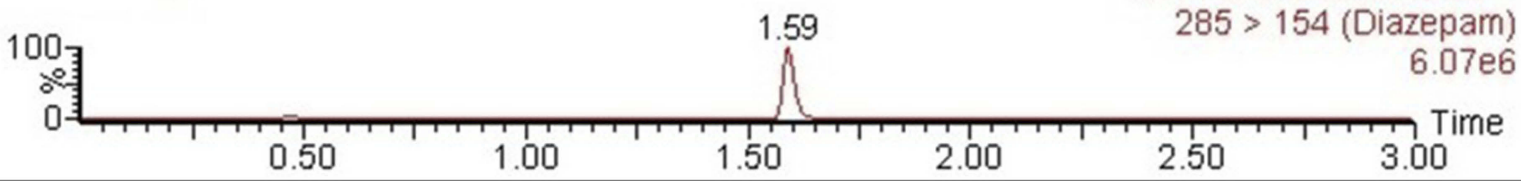

Figure 2 Representative chromatograms of blank plasma (A), blank plasma spiked with standard solution (B), and plasma sample obtained from oral administration of fruquintinib in rats (C). 
Table I The precision, accuracy, and extraction efficiency of fruquintinib $(n=6)$

\begin{tabular}{|l|l|l|l|l|l|}
\hline \multirow{2}{*}{ Concentration $(\mathbf{n g} / \mathbf{m L})$} & \multicolumn{2}{|l|}{ Precision (RSD \%) } & \multicolumn{2}{l|}{ Accuracy (RE \%) } & \multirow{2}{*}{ Extraction efficiency (\%) mean \pm RSD } \\
\cline { 2 - 5 } & Intraday & Interday & Intraday & Interday & \\
\hline 2 & 11.9 & 9.8 & -13.0 & -7.0 & $85.2 \pm 13.1$ \\
80 & 6.6 & 2.5 & 11.7 & 13.7 & $94.4 \pm 4.2$ \\
800 & 5.8 & 2.3 & -5.1 & -3.0 & $82.0 \pm 3.5$ \\
\hline
\end{tabular}

Abbreviations: RSD, relative standard deviation; RE, relative error.

Table 2 Stability results of fruquintinib in rat plasma in different conditions $(n=5)$

\begin{tabular}{|l|l|l|l|l|l|l|l|l|}
\hline \multirow{2}{*}{ Concentration (ng/mL) } & \multicolumn{2}{l|}{ Room temperature, I 2 hrs } & \multicolumn{2}{l|}{$\mathbf{4}^{\circ} \mathbf{C}, \mathbf{l} 2$ hrs } & \multicolumn{2}{l|}{ Three freeze-thaw } & \multicolumn{2}{l|}{$-80^{\circ} \mathbf{C}, 28$ days } \\
\cline { 2 - 9 } & RSD (\%) & RE (\%) & RSD (\%) & RE (\%) & RSD (\%) & RE (\%) & RSD (\%) & RE (\%) \\
\hline 2 & 12.12 & 3.91 & 9.10 & 7.10 & 11.81 & -2.38 & 6.20 & -2.38 \\
80 & 4.29 & -1.67 & 4.19 & -4.21 & 14.00 & 4.66 & 11.91 & 8.42 \\
800 & 1.95 & 2.32 & 0.74 & 6.14 & 9.34 & 5.16 & 8.92 & 3.63 \\
\hline
\end{tabular}

Abbreviations: RSD, relative standard deviation; RE, relative error.

Table 3 The pharmacokinetic parameters of fruquintinib in rat plasma after oral administration of $1.0 \mathrm{mg} / \mathrm{kg}$ fruquintinib $(n=8$, mean $\pm \mathrm{SD}$ )

\begin{tabular}{|l|l|}
\hline Parameters & Fruquintinib \\
\hline$t_{1 / 2}(\mathrm{hrs})$ & $3.34 \pm 1.04$ \\
$T_{\max }(\mathrm{hrs})$ & $4.25 \pm 1.17$ \\
$\mathrm{CL} / \mathrm{F}(\mathrm{L} / \mathrm{h} / \mathrm{kg})$ & $0.21 \pm 0.12$ \\
$C_{\max }(\mathrm{ng} / \mathrm{mL})$ & $712.28 \pm 319.40$ \\
$\mathrm{AUC}_{0-\mathrm{t}}(\mathrm{ng} / \mathrm{mL} \cdot \mathrm{h})$ & $6267.54 \pm 3004.66$ \\
$\mathrm{AUC}_{0 \rightarrow \infty}(\mathrm{ng} / \mathrm{mL} \cdot \mathrm{h})$ & $6269.42 \pm 3005.53$ \\
\hline
\end{tabular}

Abbreviations: $t_{1 / 2}$, half-life; $T_{\max }$, time to reach maximum plasma concentration; CL/F, plasma clearance rate; $C_{\max }$, the maximum plasma concentration; $\mathrm{AUC}_{0-\mathrm{t}}$, area under the systemic drug concentration-time curve from time 0 to $\mathrm{t} ; \mathrm{AUC}_{0 \rightarrow \infty}$, area under the systemic drug concentration-time curve from time 0 to infinity.

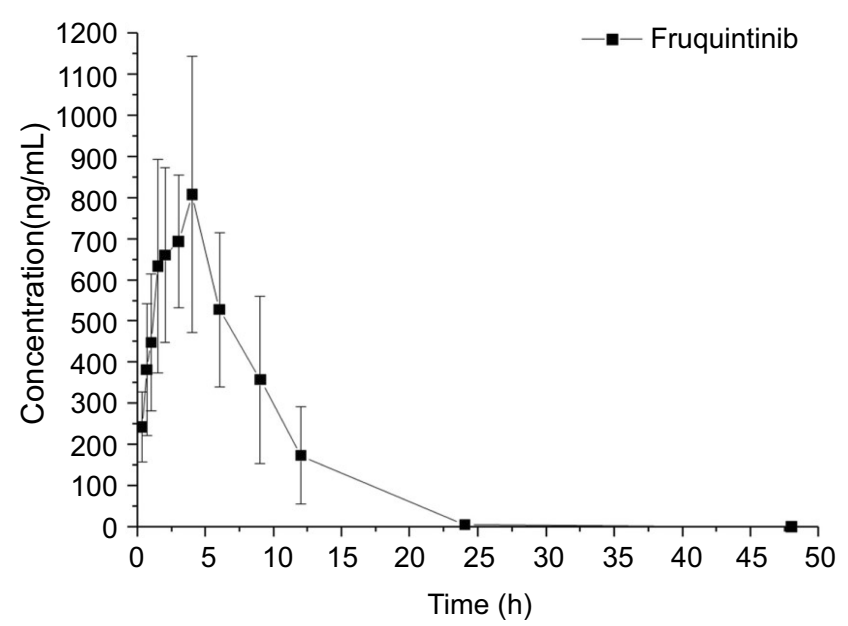

Figure 3 Mean plasma concentration-time profiles of fruquintinib in 8 rats (mean $\pm S D$ ) after oral administration of $1.0 \mathrm{mg} / \mathrm{kg}$ fruquintinib. rat plasma and then applied to the pharmacokinetic study. This method covers a wide range of the concentration curve from 1.0 to $1000 \mathrm{ng} / \mathrm{mL}$ for fruquintinib. With small sample volume, simple extraction procedure, and fast analysis, this analytical method is applicable for large studies. It would help to elucidate the potential pharmacological actions of fruquintinib through the pharmacokinetic results.

\section{Abbreviations}

UPLC-MS/MS, ultraperformance liquid chromatography-tandem mass spectrometry; LLOQ, lower limit of quantification; VEGF, vascular endothelial growth factor; VEGFR, vascular endothelial growth factor receptor; CFDA, China Food and Drug Administration; NSCLC, non-small-cell lung cancer; CRC, colorectal cancer; QCs, quality control sample; LCMS/MS, liquid chromatography-tandem mass spectrometry; $\mathrm{m} / \mathrm{z}$, precursor-to-product ion transitions of fruquintinib.

\section{Acknowledgment}

This work was supported by the First Affiliated Hospital of Wenzhou Medical University (FHY2015019).

\section{Disclosure}

The authors report no conflicts of interest in this work.

\section{References}

1. Xu RH, Li J, Bai Y, et al. Safety and efficacy of fruquintinib in patients with previously treated metastatic colorectal cancer: a phase Ib study and a randomized double-blind phase II study. J Hematol Oncol. 2017;10(1):22-30. doi:10.1186/s13045-016-0384-9 
2. Cao J, Zhang J, Peng W, et al. A Phase I study of safety and pharmacokinetics of fruquintinib, a novel selective inhibitor of vascular endothelial growth factor receptor-1, -2 , and -3 tyrosine kinases in Chinese patients with advanced solid tumors. Cancer Chemother Pharmacol. 2016;78(2):259-269. doi:10.1007/s00280-016-3069-8

3. Boere IA, Hamberg P, Sleijfer S. It takes two to tango: combinations of conventional cytotoxics with compounds targeting the vascular endothelial growth factor-vascular endothelial growth factor receptor pathway in patients with solid malignancies. Cancer Sci. 2010;101 (1):7-15. doi:10.1111/j.1349-7006.2009.01369.x

4. Veeravagu A, Hsu AR, Cai W, Hou LC, Tse VC, Chen X. Vascular endothelial growth factor and vascular endothelial growth factor receptor inhibitors as anti-angiogenic agents in cancer therapy. Recent Pat Anticancer Drug Discov. 2007;2(1):59-71.

5. Sun Q, Zhou J, Zhang Z, et al. Discovery of fruquintinib, a potent and highly selective small molecule inhibitor of VEGFR 1, 2, 3 tyrosine kinases for cancer therapy. Cancer Biol Ther. 2014;15(12):16351645. doi:10.4161/15384047.2014.964087

6. Zhou CC, Bai CX, Guan ZZ, et al. Safety and efficacy of first-line bevacizumab combination therapy in Chinese population with advanced non-squamous NSCLC: data of subgroup analyses from MO19390 (SAiL) study. Clin Transl Oncol. 2014;16(5):463-468. doi:10.1007/s12094-013-1102-5

7. Grandinetti CA, Goldspiel BR. Sorafenib and sunitinib: novel targeted therapies for renal cell cancer. Pharmacotherapy. 2007;27 (8):1125-1144. doi:10.1592/phco.27.8.1125

8. Mross K, Frost A, Steinbild S, et al. A phase I dose-escalation study of regorafenib (BAY 73-4506), an inhibitor of oncogenic, angiogenic, and stromal kinases, in patients with advanced solid tumors. Clin Cancer Res. 2012;18(9):2658-2667. doi:10.1158/1078-0432.CCR-11-1900

9. Sartore-Bianchi A, Zeppellini A, Amatu A, Ricotta R, Bencardino K, Siena S. Regorafenib in metastatic colorectal cancer. Expert Rev Anticancer Ther. 2014;14(3):255-265. doi:10.1586/14737140. 2014.894887

10. Keating GM, Santoro A. Sorafenib: a review of its use in advanced hepatocellular carcinoma. Drugs. 2009;69(2):223-240. doi:10.2165/ 00003495-200969020-00006

11. van Geel RM, Beijnen JH, Schellens JH. Concise drug review: pazopanib and axitinib. Oncologist. 2012;17(8):1081-1089. doi:10.1634/theoncologist.2012-0055

12. Nosov DA, Esteves B, Lipatov ON, et al. Antitumor activity and safety of tivozanib (AV-951) in a phase II randomized discontinuation trial in patients with renal cell carcinoma. J Clin Oncol. 2012;30 (14):1678-1685. doi:10.1200/JCO.2011.35.3524

13. Rugo HS, Herbst RS, Liu G, et al. Phase I trial of the oral antiangiogenesis agent AG-013736 in patients with advanced solid tumors: pharmacokinetic and clinical results. $J$ Clin Oncol. 2005;23 (24):5474-5483. doi:10.1200/JCO.2005.04.192

14. Liang S, Xu Y, Tan F, Ding L, Ma Y, Wang M. Efficacy of icotinib in advanced lung squamous cell carcinoma. Cancer Med. 2018. doi:10.1002/cam4.1736

15. Bao SS, Wen J, Zheng X, et al. Evaluation of the inhibition effects of apatinib on human and rat cytochrome P450. Toxicol Lett. 2018. doi:10.1016/j.toxlet.2018.08.010
16. Liu S, Wei H, Gong X, et al. LC-MS/MS method for simultaneous determination of famitinib and its major metabolites in human plasma. Bioanalysis. 2018;10(11):791-801. doi:10.4155/bio-2017-0258

17. Jiang JF, Chen XY, Zhong DF. Metabolic research of domestically developed small molecule tyrosine kinase inhibitors. Yao Xue Xue Bao. 2016;51(2):248-256.

18. Silva-Oliveira RJ, Silva VA, Martinho O, et al. Cytotoxicity of allitinib, an irreversible anti-EGFR agent, in a large panel of human cancer-derived cell lines: KRAS mutation status as a predictive biomarker. Cell Oncol (Dordr). 2016;39(3):253-263. doi:10.1007/ s13402-016-0270-z

19. Li J, Qin S, Xu RH, et al. Effect of fruquintinib vs placebo on overall survival in patients with previously treated metastatic colorectal cancer: the FRESCO randomized clinical trial. Jama. 2018;319 (24):2486-2496. doi:10.1001/jama.2018.7855

20. Zhang L, Fan C, Guo Z, et al. Discovery of a potent dual EGFR/ HER-2 inhibitor L-2 (selatinib) for the treatment of cancer. Eur $J$ Med Chem. 2013;69:833-841. doi:10.1016/j.ejmech.2013.09.032

21. Zhou S, Shao F, Xu Z, et al. A phase I study to investigate the metabolism, excretion, and pharmacokinetics of [(14)C]fruquintinib, a novel oral selective VEGFR inhibitor, in healthy Chinese male volunteers. Cancer Chemother Pharmacol. 2017;80(3):563-573. doi:10.1007/s00280-017-3394-6

22. Cassidy S, Syed BA. Colorectal cancer drugs market. Nat Rev Drug Discov. 2017;16(8):525-526. doi:10.1038/nrd.2017.59

23. Ren Y, Sun Q, Long J, et al. Evaluation of fruquintinib, a potent and selective oral VEGFR inhibitor, in combination with targeted therapies or immune checkpoint inhibitors in preclinical tumor models. Cancer Research. 2017;77(13):19106-14404.

24. Burki TK. Fruquintinib for previously treated metastatic colorectal cancer. Lancet Oncol. 2018;19(8):e388.

25. Gu Y, Wang J, Li K, et al. Preclinical pharmacokinetics and disposition of a novel selective VEGFR inhibitor fruquintinib (HMPL-013) and the prediction of its human pharmacokinetics. Cancer Chemother Pharmacol. 2014;74(1):95-115. doi:10.1007/s00280-014-2471-3

26. Health UDo, Human services F, Drug Administration CfDE, Research CfVm. Guidance for industry, bioanalytical method validation. Fed Regist. 2001;66(4):206-207.

27. Yun C, Ding L, Leng Y, Zhu H, Wen A, Yang L. Determination of ipriflavone in human plasma by LC-MS and its application in a pharmacokinetic study. Biomed Chromatogr. 2012;26(1):123-128. doi:10.1002/bmc.1641

28. Yun C, Dashwood WM, Kwong LN, et al. Accurate quantification of PGE2 in the polyposis in rat colon (Pirc) model by surrogate analytebased UPLC-MS/MS. J Pharm Biomed Anal. 2018;148:42-50. doi:10.1016/j.jpba.2017.07.025

29. Yun C, Yin T, Shatzer K, et al. Determination of 7alpha-OH cholesterol by LC-MS/MS: application in assessing the activity of CYP7A1 in cholestatic minipigs. J Chromatogr B Analyt Technol Biomed Life Sci. 2016;1025:76-82. doi:10.1016/j.jchromb.2016.05.005

30. Xu RA, Lin Q, Qiu X, et al. UPLC-MS/MS method for the simultaneous determination of imatinib, voriconazole and their metabolites concentrations in rat plasma. J Pharm Biomed Anal. 2019;166:6-12. doi:10.1016/j.jpba.2018.12.036

Drug Design, Development and Therapy

Dovepress

\section{Publish your work in this journal}

Drug Design, Development and Therapy is an international, peerreviewed open-access journal that spans the spectrum of drug design and development through to clinical applications. Clinical outcomes, patient safety, and programs for the development and effective, safe, and sustained use of medicines are a feature of the journal, which has also

been accepted for indexing on PubMed Central. The manuscript management system is completely online and includes a very quick and fair peer-review system, which is all easy to use. Visit http://www. dovepress.com/testimonials.php to read real quotes from published authors. 